\title{
Amisulpride en el tratamiento de la dependencia alcohólica
}

\section{Amisulpride for the treatment of alcohol dependence}

\author{
Gerardo Flórez ; Pllar A Salz $z^{\star *}$ \\ Paz García-Portilla ${ }^{* *}$; Sandra Álvarez ${ }^{*}$; \\ LuIS NogueIRAS*; JuLIO BobeS ${ }^{\star \star}$
}

\author{
Unidad de Conductas Adictivas, Servicio Gallego de Salus, \\ Ourense, España. \\ ** Departamento de Psichiatría, Universidad de Oviedo. \\ Centro de Investigación Biomédica en Red de Salud Mental, \\ CIBERSAM, Oviedo, España.
}

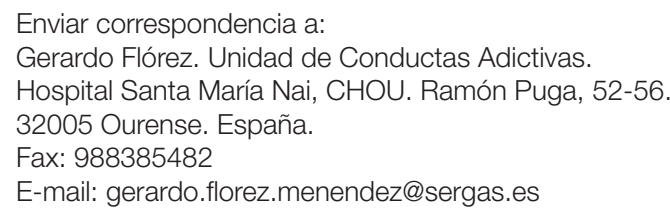

E-mail: gerardo.florez.menendez@sergas.es

\section{RESUMEN}

Objetivos: Estudio abierto, naturalístico de 6 meses de seguimiento, para comparar la eficacia de amisulpride frente a topiramato y naltrexona en pacientes con dependencia al alcohol. Métodos: se han incluido un total de 274 pacientes diagnosticados de dependencia al alcohol y con un consumo intenso de alcohol durante el último mes. Una vez desintoxicados los pacientes fueron asignados a diferentes grupos de tratamiento (naltrexona a $50 \mathrm{mgr}$ por día, topiramato a $200 \mathrm{mgr}$ por día 0 amisulpride a $100 \mathrm{mgr}$ por día). Dichos pacientes fueron evaluados al inicio del tratamiento y a los 3 y 6 meses de seguimiento mediante instrumentos para medir el consumo de alcohol (EuropASI y Alcohol Timeline Followback), el craving (OCDS), la discapacidad (WHO/DAS) y la calidad de vida (EQ-5D); también se utilizaron marcadores biológicos de consumo de alcohol. Resultados: a los 6 meses los pacientes que tomaban amisulpride obtenían peores resultados en variables relacionadas directamente con el consumo de alcohol (OCDS, alcohol consumido, número de bebidas por día de consumo y días de consumo intenso), sin embargo no se encontraron diferencias significativas en esas variables al compararse con naltrexona. Conclusiones: en este estudio, amisulpride, a dosis de $100 \mathrm{mgr}$ por dia, fue menos eficaz que topiramato, a dosis de $200 \mathrm{mgr}$ por día, pero demostró eficacia similar a la naltrexona, a dosis de $50 \mathrm{mgr}$ por día, a la hora de reducir el consumo de alcohol y el craving.

Palabras clave: Amisulpride, naltrexona, topiramato, dependencia alcohólica y tratamiento. recibido: iunio 2010 aceptado: enero 2011

\section{ABSTRACT}

Background: 6-month naturalistic, open-label trial to compare amisulpride versus topiramate and naltrexone as a treatment for patients with alcohol dependence, with assessments at enrolment and after 3 and 6 months of treatment. Methods: 274 alcohol-dependent patients who had been drinking heavily during the past month were included. Once detoxified, patients were assigned to one of three treatment groups (naltrexone $50 \mathrm{mgr}$ per day, topiramate $200 \mathrm{mgr}$ per day or amisulpride $100 \mathrm{mgr}$ per day). Patients were assessed at baseline and after 3 and 6 months of follow-up. Outcome was measured using tools that assessed alcohol intake (EuropASI and Alcohol Timeline Followback), craving (OCDS), disability (WHO/DAS), and quality of life (EO-5D); changes in biomarkers of alcohol intake were also noted. Results: at the 6-month follow-up patients taking amisulpride had poorer results than those taking topiramate in direct measures of alcohol intake (OCDS, alcohol intake, number of drinks per day and heavy drinking days), but no significant differences were found in these measures on comparing the amisulpride patients with those taking naltrexone. Conclusions: in this study, amisulpride, at a dose of $100 \mathrm{mgr}$ per day, was less effective than topiramate, at a dose of $200 \mathrm{mg}$ per day, but as effective as naltrexone, at a dose of $50 \mathrm{mg}$ per day, for reducing alcohol intake and craving over the period of the study.

Key words: Amisulpride, naltrexone, topiramate, alcohol dependence and treatment. 


\section{INTRODUCCIÓN}

E etanol es un agente psicoactivo adictivo peculiar en lo referente a su actividad en el cerebro humano. Al contrario que otros agentes psicoactivos adictivos, como la heroína o la nicotina, que tienen una única diana en los sistemas de neurotransmisión cerebral, el etanol modifica de forma directa la actividad de numerosos neurotransmisores ${ }^{1}$ : Su consumo agudo estimula la actividad serotoninérgica a través del receptor 5 HT-3, estimula la actividad colinérgica a través de receptores nicotínicos, incrementa la actividad gabaérgica a través del receptor GABA A, inhibe la actividad glutamatérgica a través de los receptores NMDA y estímula la actividad opioide a través del receptor $\mu$. Si el consumo de alcohol persiste de forma crónica se produce un estado de tolerancia molecular en el que el cerebro disminuye su actividad gabaérgica intrínseca e incrementa su actividad glutamatérgica intrínseca, por otro lado se produce una hipoactividad dopaminérgica y serotoninérgica; este escenario lleva al consumidor a beber alcohol de forma fásica, pero crónica, para mantener un equilibrio en su cerebro "adaptado" a la presencia de concentraciones importantes de etanol. Esta intensa modificación en los sistemas de neurotransmisión determina que el paciente dependiente al alcohol deba enfrentarse durante la fase de deshabituación a un deseo de consumo de alcohol mediado por las vías neurobiológicas del "craving" que implican un descenso en la actividad de los sistemas mediadores de la recompensa - desequilibrio dopaminérgico producido indirectamente por las alteraciones opioides y gabaérgicas principalmente - y por la impulsividad e inestabilidad emocional que implica una hiperactivación de los sistemas cerebrales que median el refuerzo negativo y la respuesta al estrés- mediadas por el desequilibrio gabaérgico / glutamatérgico ${ }^{2}$.

Numerosos tratamientos psicofarmacológicos han sido ensayados para corregir estas alteraciones en el funcionamiento cerebral y mejorar las tasas de abstinencia. Más allá de aquellos tratamientos que tienen la indicación - naltrexo$n^{3}{ }^{3}$, acamprosato ${ }^{4}$ y disulfiram ${ }^{5}$ - los resultados obtenidos por otras moléculas han sido erráticos. El anticonvulsivante topiramato, un regulador gabaérgico / glutamatérgico, ha obtenido evidencias de eficacia a través de dos estudios controlados $^{6,7}$. Varios agentes antipsicóticos, moduladores de las vías dopaminérgicas, también han sido ensayados con resultados dispares y con poca evidencia de eficacia hasta la fecha ${ }^{8,9}$.

El objetivo de este estudio es comparar la eficacia en la deshabituación alcohólica del antipsicótico amisulpride, con el antagonista opioide naltrexona y con el anticonvulsivante topiramato.

\section{MÉTODOS}

Estudio naturalístico, abierto, en el que se compara la eficacia de amisulpride en comparación con naltrexona y con topiramato para la deshabituación alcohólica.

\section{Pacientes}

El estudio se realizó en un dispositivo de tratamiento ambulatorio integrado en la red pública del SERGAS.

Los criterios de inclusión fueron: (1) cumplir criterios para un diagnóstico de dependencia al alcohol según la Clasificación Internacional de Enfermedades Décima Edición (CIE - 10) ${ }^{10}$, (2) tener una ingesta de etanol en los seis meses previos al inicio del tratamiento de al menos 210 gramos a la semana en el caso de los varones y de al menos 140 gramos en el caso de las mujeres, - estos son los límites para el consumo de bajo riesgo según la Organización Mundial de la Salud ${ }^{11}$-, evaluado utilizando el European Addiction Severity Index (EuropASI) ${ }^{12}$ y el Alcohol Timeline Followback ${ }^{13}$, (3) manifestar el deseo de dejar de beber alcohol.

Los criterios de exclusión fueron: (1) mayores de 65 años o menores de 18 años, (2) presencia de una dependencia a otra sustancia de abuso que no fuera la nicotina, valorado con el EuropASI y un control de orina, (3) Presencia de un trastorno psiquiátrico que no fuera un Trastorno de la Personalidad, valorado con el EuropASI, (4) presencia de una enfermedad orgánica que en opinión de los investigadores afectase de forma negativa a la participación del paciente en el estudio, valorado con el EuropASI, (5) incapacidad para firmar el consentimiento informado completo, (6) incapacidad para expresarse correctamente en Castellano o en Gallego, (7) historia de retraso mental, (8) embarazo o lactancia, y (9) ausencia de informador significativo, situación familiar o social estable, que pudiese informar a los investigadores del consumo diario del paciente.

Fueron valorados, para su inclusión el grupo de Amisulpride, todos los pacientes que acudieron buscando tratamiento para su dependencia al alcohol entre Mayo del 2008 y Mayo del 2009. De estos 348 pacientes, 95 cumplian los criterios de inclusión y exclusión, de ellos 3 rechazaron participar en el estudio, siendo incluidos los 92 restantes que firmaron el consentimiento informado. La selección de los grupos naltrexona y topiramato se realizó previamente tal y como se detalla en una publicación previa ${ }^{14}$.

\section{Tratamiento}

Los pacientes fueron desintoxicados con clorazepato antes de iniciar el tratamiento de deshabituación ${ }^{15}$. A continuación los pacientes tomaron $100 \mathrm{mgr}$ de amisulpride por día, se escogió esta dosis ya que en el ensayo clínico realizado previamente con Amisulpride la dosis de $50 \mathrm{mgr}$ por día no demostró ser superior a placebo ${ }^{9}$. La evolución de estos pacientes fue comparada con la del grupo a tratamiento con naltrexona, 91 pacientes con dosis de 50 mgr por dia, y topiramato, 91 pacientes con dosis de 200 mgr por día ${ }^{14}$.

Cuando los pacientes no experimentaban una evolución favorable, abstinencia o consumo moderado sin problemas, se añadía si el paciente así lo aceptaba disulfiram a dosis de 250 mgr por día. Automáticamente se consideraba un fracaso del tratamiento deshabituador y se analizaba como tal. Los pacientes consultaban con el psiquiatra de la unidad al menos una vez al mes para revisar su tratamiento. 
Todos los pacientes recibieron sesiones semanales, durante las cuales se valoraba el consumo de alcohol, de una intervención psicosocial denominada BRENDA, esta intervención está protocolizada y estructurada ${ }^{16}$. La intervención fue llevada a cabo por psicólogos clínicos formados en el modelo.

\section{Evaluación}

Los pacientes fueron evaluados al inicio del tratamiento deshabituador y 3 y 6 meses después de haber iniciado dicho tratamiento.

Evaluación basal: (1) EuropASI ${ }^{12}$ para valorar el consumo de alcohol y sus consecuencias; (2) Alcohol Timeline Followback ${ }^{13}$ para valorar el consumo de alcohol; (3) Obsessive Compulsive Drinking Scale ${ }^{17}$ (OCDS) para valorar el Craving alcohólico; (4) Fagerström Test ${ }^{18}$ para valorar la dependencia nicotínica; (5) International Personality Disorder Examination $^{19}$ (IPDE) para valorar la presencia de Trastornos de la Personalidad; (6) Readiness to Change Questionnaire ${ }^{20}$ (RCO) para valorar la motivación para dejar de consumir alcohol; (7) WHO Psychiatric Disability Assessment Schedule ${ }^{21}$ (WHO/ DAS) para valorar discapacidad producida por el consumo de alcohol; (8) European Quality of Life Questionnaire ${ }^{22}$ (EQ-5D) para valorar la calidad de vida. La valoración inicial también incluía marcadores biológicos relacionados con el consumo excesivo de alcohol ${ }^{23}$ : gammaglutamiltransferasa (GGT), aspartatoaminotransferasa (ASAT/GOT), alaninoaminotransferasa (ALAT/GPT) y el volumen corpuscular medio (VCM), y el ratio ASAT/ALAT.

Evaluación a los 3 y 6 meses: (1) EuropASI; (2) OCDS; (3) Fagerström; (4) WHO/DAS; (5) EQ-5D; (6) Alcohol Timeline Followback. A los 6 meses se realizaron de nuevo los marcadores biológicos comentados previamente.

Cada mes la tolerancia al tratamiento fue evaluada usando la UKU Side Effect Rating Scale ${ }^{24}$. Si algún efecto secundario alcanzaba la puntuación de 3 el tratamiento se reducía a la mitad.

Mensualmente se valoró la adherencia al tratamiento utilizando el test de Morisky-Green ${ }^{25}$, se entrevistó al paciente y al informador significativo para luego utilizar la puntuación inferior. También se realizó mensualmente un recuento de comprimidos.

El consumo de alcohol se valoró en cada sesión de tratamiento. Se valoraron los consumos según la información del paciente y del informador significativo utilizando siempre el valor más elevado.

Los abandonos se definieron como interrupción de las visitas durante al menos un mes. Para reducir la tasa de abandono se contactó telefónicamente con el paciente que no acudía a una consulta o con su informador significativo.

\section{Eficacia}

La buena evolución de los pacientes no sólo se objetivó con los instrumentos mencionados anteriormente, también se construyó una escala de evolución, utilizando las reco- mendaciones de los investigadores de los estudios MATCH y COMBINE $26,27,28,29$, con los siguientes grupos de evolución:

1. Abstinencia: No se informa de consumo de alcohol en los tres meses previos.

2. Consumo moderado sin problemas: Se informa de un consumo inferior a 40 gramos de etanol por día en hombres e inferior a 30 gramos en mujeres, no se informa de problemas derivados de ese consumo (puntuaciones de 4 o menos en el EuropASI).

3. Consumo moderado con problemas: Como el anterior pero hay puntuaciones superiores a 4 en alguna escala del EuropASI.

4. Consumo excesivo sin problemas: Consumo superior al moderado sin problemas derivados del mismo.

5. Consumo excesivo con problemas: Consumo superior al moderado con problemas derivados del mismo (puntuaciones de 4 o más en el EuropASI).

Los pacientes en los grupos 3,4,5 y los abandonos fueron considerados como mala evolución.

También se valoró la evolución de variables relacionadas con el consumo de alcohol, utilizadas en estudios previos ${ }^{6,29}$, usando el Alcohol Timeline Followback: Número de días de "consumo intenso" donde hubo consumos superiores a 60 gramos de etanol, porcentaje de días de abstinencia, dias totales de consumo de alcohol, dias hasta el primer consumo de alcohol y consumiciones medias (10 gramos de etanol) por día de consumo.

\section{Análisis estadísticos}

Los datos de todos los pacientes fueron analizados por intención de tratar. La calidad de la recogida de datos fue garantizada por un coordinador externo al proyecto que revisó la inclusión de todos los datos a la búsqueda de datos inusuales o incompletos.

Se estudió las diferencias entre cada tratamiento en cada punto de corte pero también los cambios longitudinales que se produjeron en los instrumentos.

Los grupos de evolución se midieron de dos formas diferentes. Primero abstinentes frente al resto y luego abstinentes mas consumo moderado sin problemas frente al resto.

Seguidamente se procedió con el análisis descriptivo de las variables de estudio. Para todas las variables se determinó el índice de asimetría, todas ellas son simétricas y por ello se utilizó la media como indice representativo, con la excepción de las variables biológicas donde se utilizó la mediana y el rango. En el caso de variables nominales se recogió el número de casos en cada una de las categorías, reflejando el porcentaje que representaron con respecto al total, y enunciándolo en una tabla de frecuencias.

Seguidamente se procedió con el análisis descriptivo de las variables de estudio. Los datos estadísticos descriptivos de las variables numéricas se expresaron como media \pm desviación estándar, valor máximo y valor mínimo. En el caso de 
variables nominales se recogió el número de casos en cada una de las categorías, reflejando el porcentaje que representaron con respecto al total, y enunciándolo en una tabla de frecuencias.

El estudio estadístico comparativo de los datos numéricos se llevó a cabo mediante análisis de la varianza de un solo factor (ANOVA) y test de HSD de Tukey para las comparativas múltiples, antes de aplicar el test se comprobó si los datos seguían una distribución normal y la igualdad de las varianzas. En aquellos casos en los que no se cumplieron los criterios de homogeneidad de las varianzas, se aplicó el test de Brown-Forsythe para los análisis de las varianzas y el test de Games-Howell para las comparativas múltiples. Las variables categóricas se analizaron mediante la prueba ji cuadrado (2).

También se ha realizado el estudio estadístico mediante análisis de la varianza con medidas repetidas (MANOVA), previamente se realizaron pruebas de elasticidad y de esfericidad. No se encontraron diferencias significativas en su comparación con el ANOVA.

Utilizando el género como covariante en este estudio no se han encontrado diferencias significativas.

Para conocer el tiempo de abstinencia hasta el primer consumo de cada tratamiento se empleó el método de Kaplan-Meier y las curvas de supervivencia obtenidas se compararon utilizando el test log rank.

Todos los análisis estadísticos fueron realizados por Trabeculae ${ }^{\circledR}$, Empresa de Base Tecnológica S.L., utilizando el Statistical Package for Social Sciences (SPSS Inc., Chicago, IL), v. 17.0 para Windows. Una $p<0.05$ fue considerada estadisticamente significativa.

\section{RESULTADOS}

A nivel basal los tres grupos no presentaban diferencias significativas en variables sociodemográficas, en cuanto a las variables clínicas y relacionadas se observaron las siguientes diferencias significativas:

(1) Topiramato versus amisulpride: OCDS obsesivo (6.18 vs 8.12 , Diferencia de Medias $(D M)=1.943$, Error Típico (ET) $=0.674, p=0.012)$, OCDS total (17.45 vs 20.25, DM $=2.794$, $\mathrm{ET}=1.107, \mathrm{p}=0.032$ ) estas puntuaciones indican un mayor nivel de gravedad en los pacientes del grupo de amisulpride; EuropASI problemas médicos (6.22 vs 5.39, DM=0.828, ET= $0.333, p=0.036)$, EuropASI alcohol (7.82 vs 6.93, $D M=0.889$, $\mathrm{ET}=0.090, \mathrm{p}<0.001$ ), EuropASI familiar/social (7 vs 6.22, $\mathrm{DM}=0.782, \mathrm{ET}=0.271, \mathrm{p}=0.012)$, EuropASI psicológico (7.45 vs $6.25, \mathrm{DM}=1.200, \mathrm{ET}=0.193, \mathrm{p}<0.001$ ) estas puntuaciones indican un mayor nivel de gravedad en los pacientes del grupo de topiramato; WHO/DAS cuidado personal (1.44 vs 2.04, $\mathrm{DM}=0.603, \mathrm{ET}=0.218, \mathrm{p}=0.018)$, WHO/DAS funcionamento familiar (3.64 vs 3.26, $\mathrm{DM}=0.376, \mathrm{ET}=0.165, \mathrm{p}=$ 0.062), WHO/DAS funcionamiento en el contexto social (3.42 vs $2.88, \mathrm{DM}=0.537, \mathrm{ET}=0.161, \mathrm{p}=0.03$ ) estas puntuaciones indican un resultado mixto en nivel de gravedad; cociente $\mathrm{GOT} / \mathrm{GPT}$ (1.31 vs $1.62, \mathrm{DM}=0.302, \mathrm{ET}=0.104, \mathrm{p}=0.012$ ).
Estas puntuaciones indican un mayor nivel de gravedad en los pacientes del grupo de topiramato.

(2) Naltrexona versus amisulpride: EuropASI alcohol (7.75 vs 6.93, $\mathrm{DM}=0.812, \mathrm{ET}=0.092, \mathrm{p}<0.001)$, EuropASI familiar/social (6.93 vs 6.22, $\mathrm{DM}=0.716, \mathrm{ET}=0.271, \mathrm{p}=0.024$ ), EuropASI psicológico (7.33 vs 6.25, DM=1.079, $\mathrm{ET}=0.202$, $p<0.001$ ) estas puntuaciones indican un mayor nivel de gravedad en los pacientes del grupo de naltrexona; WHO/DAS cuidado personal (1.47 vs 2.04, $\mathrm{DM}=0.570, \mathrm{ET}=0.208, \mathrm{p}=$ 0.019), WHO/DAS funcionamento familiar (3.71 vs 3.26, DM= $0.453, E T=0.165, p=0.018)$, WHO/DAS funcionamiento en el contexto social (3.38 vs 2.88, $\mathrm{DM}=0.504, \mathrm{ET}=0.157, \mathrm{p}=$ $0.05)$; Escala analógica visual EQ-5D (81.26 vs 73.20, DM= 8.057, $E T=1.594, p=0.006$ ). Estas puntuaciones indican un resultado mixto en nivel de gravedad.

A los 3 meses de evolución se encontraron las siguientes diferencias significativas en la evaluación transversal:

(1) Topiramato versus amisulpride: OCDS obsesivo (0.81 vs $2.24, \mathrm{DM}=1.430, \mathrm{ET}=0.420, \mathrm{p}=0.03)$, OCDS compulsivo (1.95 vs 3.44, $\mathrm{DM}=1.486, \mathrm{ET}=0.594, \mathrm{p}=0.036$ ), OCDS total (2.77 vs 5.68, $D M=1.486, E T=0.594, p=0.036$ ), EuropASI alcohol (0.43 vs $1.15, D M=0.716, E T=0.274, p=0.027)$ y WHO/DAS cuidado personal ( 0.15 vs $0.63, \mathrm{DM}=0.483, \mathrm{ET}=$ $0.130, p=0.01$ ) estas puntuaciones indican una mejor evolución de los pacientes del grupo de topiramato.

(2) Naltrexona versus amisulpride: No se encontraron diferencias significativas.

A los 3 meses de evolución se encontraron las siguientes diferencias significativas en la evolución longitudinal (comparación basal - 3 meses):

(1) Topiramato versus Amisulpride: EuropASI médico $(-5.05$ vs $-3.94, D M=1.107, E T=0.417, p=0.023)$, EuropASI familiar/social ( -5.65 vs $-4.34, D M=1.310, E T=0.398, p=$ 0.003 ), EuropASI psicológico ( -5.69 vs $-4, D M=1.686, E T=$ $0.369, p<0.001)$ y WHO/DAS funcionamiento en el contexto social amplio (-2.84 vs $-1.98, \mathrm{DM}=0.862, \mathrm{ET}=0.221, \mathrm{p}<$ 0.001 ) estas puntuaciones indican una mejor evolución de los pacientes del grupo de topiramato.

(2) Naltrexona versus amisulpride:WHO/DAS funcionamiento familiar (-2.84 vs $-2.16, \mathrm{DM}=0.635, \mathrm{ET}=0.143, \mathrm{p}=$ $0.014)$ y WHO/DAS funcionamiento en el contexto social amplio $(-2.52$ vs $-1.98, \mathrm{DM}=0.542, \mathrm{ET}=0.222, \mathrm{p}=0.041)$ estas puntuaciones indican una mejor evolución de los pacientes del grupo de naltrexona.

A los 6 meses de evolución se encontraron las siguientes diferencias significativas en la evaluación transversal:

(1) Topiramato versus amisulpride: OCDS obsesivo (0.74 vs $2.70, \mathrm{DM}=1.954, \mathrm{ET}=0.466, \mathrm{p}<0.001)$, OCDS Compulsivo (2.13 vs $4.38, \mathrm{DM}=2.249, \mathrm{ET}=0.703, \mathrm{p}=0.005)$, OCDS total (2.87 vs 7.07, $\mathrm{DM}=4.203, \mathrm{ET}=1.113, \mathrm{p}=0.001)$, EuropASI médico (0.71 vs 1.84, $\mathrm{DM}=1.136, \mathrm{ET}=0.322, \mathrm{p}=0.002)$, EuropASI empleo/recursos (0.39 vs $1.52, \mathrm{DM}=1.136, \mathrm{ET}=$ $0.303, p=0.001$ ), EuropASI alcohol ( 1.28 vs 2.55, $\mathrm{DM}=$ 1.266, $E T=0.389, p=0.004$ ), EuropASI familia/social (0.94 vs 2.35, $\mathrm{DM}=1.412, \mathrm{ET}=0.402, \mathrm{p}=0.002$ ), EuropASI psicológico 
(1.01 vs 2.45, $\mathrm{DM}=1.439, \mathrm{ET}=0.392, \mathrm{p}=0.001)$, WHO/DAS cuidado personal (0.18 vs $0.84, \mathrm{DM}=0.665, \mathrm{ET}=0.157, \mathrm{p}<$ 0.001), WHO/DAS funcionamiento ocupacional (0.27 vs 0.94 , $\mathrm{DM}=0.668, \mathrm{ET}=0.186, \mathrm{p}=0.001), \mathrm{WHO} / \mathrm{DAS}$ funcionamiento familiar (0.58 vs $1.30, D M=0.728, E T=0.231, p=0.06$ ), WHO/DAS funcionamiento en el contexto social amplio (0.46 vs 1.06, $\mathrm{DM}=0.602, \mathrm{ET}=0.203, \mathrm{p}=0.010), \mathrm{GOT}$ (31.82 vs $42.45, \mathrm{DM}=10.631, \mathrm{ET}=4.051, \mathrm{p}=0.025$ ), GGT (49.95 vs 90.48, $\mathrm{DM}=48.443, \mathrm{ET}=18.143, \mathrm{p}=0.024)$, Escala Analógica Visual del EQ-5D (96.47 vs 89.63, $\mathrm{DM}=6.836, \mathrm{ET}=2.591, \mathrm{p}=$ 0.025 ) y $\mathrm{GOT} / \mathrm{GPT}$ (1.14 vs $1.36, \mathrm{DM}=0.213, \mathrm{ET}=0.626, \mathrm{p}=$ 0.002). Estas puntuaciones indican una mejor evolución de los pacientes del grupo de topiramato.

(2) Naltrexona versus amisulpride: EuropASI empleo/ recursos (0.56 vs 1.52, $\mathrm{DM}=0.962, \mathrm{ET}=0.320, \mathrm{p}=0.009$ ), WHO/DAS cuidado personal (0.26 vs $0.84, \mathrm{DM}=0.579, \mathrm{ET}=$ $0.158, p=0.001)$, WHO/DAS funcionamiento ocupacional (0.35 vs 0.94, $\mathrm{DM}=0.589, \mathrm{ET}=0.189, \mathrm{p}=0.006$ ), Escala Ana- lógica Visual del EQ-5D (96.63 vs 89.63, DM=6.991, ET= $2.150, p=0.004)$ y $\mathrm{GOT} / \mathrm{GPT}$ ( 1.19 vs $1.36, \mathrm{DM}=0.160, \mathrm{ET}=$ $0.635, p=0.032)$. ). Estas puntuaciones indican una mejor evolución de los pacientes del grupo de naltrexona.

A los 6 meses de evolución se encontraron las siguientes diferencias significativas en la evolución longitudinal (comparación 3 meses -6 meses):

(1) Topiramato versus amisulpride: EuropASI médico $(-0.49$ vs $0.39, \mathrm{DM}=0.884, \mathrm{ET}=0.337, \mathrm{p}=0.026)$ y EuropASI psicológico ( -0.05 vs $0.38, D M=1.022, E T=0.376, p=$ 0.02). Estas puntuaciones indican una mejor evolución de los pacientes del grupo de topiramato.

(2) Naltrexona versus amisulpride: No se encontraron diferencias significativas.

En la siguiente tabla se presentan los resultados obtenidos por cada tratamiento a 3 y 6 meses de seguimiento para los grupos de consumo.

Tabla 1. Grupos de consumo para cada tratamiento

\begin{tabular}{l|c|c|c|c|c|c}
\hline \multicolumn{2}{c}{} & \multicolumn{2}{c|}{ Naltrexona } & \multicolumn{2}{c}{ Amisulpride } \\
\hline & 3 meses & 6 meses & 3 meses & 6 meses & 3 meses & 6 meses \\
\hline 1 & $39(42.9 \%)$ & $38(41.8 \%)$ & $44(48.6 \%)$ & $43(47.3 \%)$ & $43(46.7 \%)$ & $35(38 \%)$ \\
\hline 2 & $19(20.9 \%)$ & $9(9.9 \%)$ & $26(28.6 \%)$ & $25(27.5 \%)$ & $20(21.7 \%)$ & $12(13 \%)$ \\
\hline 3 & $1(1.1 \%)$ & $3(3.3 \%)$ & $2(2.2 \%)$ & $3(3.3 \%)$ & $0(0 \%)$ & $2(2.2 \%)$ \\
\hline 4 & $1(1.1 \%)$ & $0(0 \%)$ & $0(0 \%)$ & $0(0 \%)$ & $0(0 \%)$ & $0(0 \%)$ \\
\hline 5 & $17(18.7 \%)$ & $17(18.7 \%)$ & $7(7.7 \%)$ & $6(6.6 \%)$ & $12(13 \%)$ & $23(25 \%)$ \\
\hline Disulfiram & $6(6.6 \%)$ & $14(14.3 \%)$ & $7(7.7 \%)$ & $8(8.8 \%)$ & $7(7.6 \%)$ & $10(10.9 \%)$ \\
\hline
\end{tabular}

Se realizaron los siguientes análisis referentes a los grupos de consumo:

(1) Topiramato versus amisulpride: (a) abstinentes (grupo 1) frente a los demás a los 3 meses y a los 6 meses: no hay diferencias significativas. (b) Abstinentes y consumo moderado sin problemas (grupo 2) frente a los demás a los 3 meses: no hay diferencias significativas; y a los 6 meses: $74.7 \%$ vs $51.1 \%, \chi^{2}=6.959, g l=1, p=0.008$.
(2) Naltrexona versus amisulpride: se realizaron los mismos análisis que con topiramato, sin encontrar ninguna diferencia significativa.

En la siguiente tabla se presentan los resultados obtenidos por cada tratamiento a 6 meses de seguimiento para las variables relacionadas con el consumo de alcohol.

Tabla 2. Medias de las variables relacionadas con el consumo de alcohol para cada tratamiento a los 6 meses.

\begin{tabular}{lccc} 
& NALTREXONA & TOPIRAMATO & AMISULPRIDE \\
\hline \% Días abstinencia & $65.20 \%$ & $65.98 \%$ & $65.07 \%$ \\
\hline Días de consumo de alcohol & 61.18 & 56.56 & 61.80 \\
\hline Días de "consumo intenso" & 13.08 & 8.04 & 16.20 \\
\hline Consumiciones medias & 4.45 & 3.21 & 4.32 \\
\hline Días hasta el primer consumo & 92.26 & 98.97 & 97.62 \\
\hline
\end{tabular}


En cuanto a los datos derivados del análisis de las variables relacionadas con el consumo de alcohol los resultados fueron:

(1) Topiramato versus amisulpride: No se encontraron diferencias significativas a los 3 y 6 meses excepto para: consumiciones medias (10 gramos de etanol) por día de consumo a los 6 meses ( 1.39 vs 2.39, $\mathrm{DM}=0.929$, $\mathrm{ET}=0.357$, $\mathrm{p}=0.027$ ) y número de días de "consumo intenso" a los 6 meses donde hubo consumos superiores a 60 gramos de etanol (3.35 vs 8.63, $\mathrm{DM}=5.281, \mathrm{ET}=0.208, \mathrm{p}=0.033$ ). Estas puntuaciones indican una mejor evolución de los pacientes del grupo de topiramato.

(2) Naltrexona versus amisulpride: No se encontraron diferencias significativas.

No se encontraron diferencias significativas entre los grupos de tratamiento en lo referente a las siguientes variables: uso de Disulfiram, abandonos y adherencia al tratamiento.

Si se encontraron diferencias significativas en lo referente a los efectos secundarios entre topiramato y amisulpride a los 3 meses: $19.78 \%$ vs $3.49 \%, \chi^{2}=8.160, g l=1, p=0.004$. Este efecto desaparecía a los 6 meses de evolución, No se encontraron diferencias significativas entre naltrexona y amisulpride.

\section{DISCUSIÓN}

En este estudio, amisulpride a dosis de 100 mgr por día fue menos eficaz en su capacidad de deshabituación alcohólica que topiramato a dosis 200 mgr por día, esto se objetivó a través de variables relacionadas directamente con el consumo de alcohol: OCDS, EuropASI alcohol, grupos de consumo a los 6 meses ( 1 y 2 frente a los demás), consumiciones medias por día a los 6 meses y días medios de consumo excesivo a los 6 meses. Sin embargo, amisulpride a estas dosis no fue superado en su eficacia deshabituadora por naltrexona a dosis de 50 mgr por día, es cierto que naltrexona obtuvo mejores resultados en algunas variables indirectas: EuropASI no alcohol, WHO/DAS y EQ-5D, pero en todas las medidas directas relacionadas con el consumo de alcohol (EuropASI alcohol, OCDS, grupos de consumo y variables relacionadas con el consumo) no se observaron diferencias significativas fueron similares en ambos grupos.

Estos resultados podrían indicar que la capacidad del topiramato de potenciar la actividad gabaérgica, antagonizar la acción glutamatérgica y modular los canales del calcio, todos ellos alterados de forma directa por el consumo crónico de etanol ${ }^{1,6,30}$, le podría dar una mayor capacidad deshabituadora frente a la capacidad de naltrexona, tal y como indicaban los resultados obtenidos en un estudio realizado con estos mismos grupos de pacientes previamente ${ }^{14}$, de inhibir la respuesta opioide del etanol, que indirectamente produciría una descarga dopaminérgica en las vías cerebrales que median la respuesta a los incentivos ${ }^{1,3,30}$, y frente a la capacidad de amisulpride a dosis bajas, menos de $200 \mathrm{mgr}$ por dia, de facilitar la transmisión dopaminérgica a través del bloqueo de receptores presinápticos lo que corregiría el deficit dopaminérgico propio de la fase de deshabituación de cualquier sustancia adictiva ${ }^{31}$, sería por lo tanto otra vía indirecta en los efectos crónicos del etanol. En resumen, el agente farmacológico (topiramato) que al antagonizar mejor los efectos que produce en el cerebro detener el consumo crónico de etanol y por lo tanto podría combatir mejor los estados mentales negativos propios de ese escenario, obtiene mejores resultados que el agente farmacológico que podría evita el refuerzo del consumo de alcohol (naltrexona), tal y como indicaban los resultados obtenidos en un estudio realizado con estos mismos grupos de pacientes previamente ${ }^{14}, y$ que el agente prohedónico que podría compensar la falta de estados cerebrales positivos durante la fase de deshabituación y con ello el riesgo de consumo para remontarlos (amisulpride) y sin riesgo de generar una nueva adicción.

No obstante, cabe destacar que los resultados positivos obtenidos en el grupo de amisulpride, con un 51\% de los pacientes a los 6 meses en grupos de consumo de buena evolución, confirma la hipótesis general de que los agentes agonistas dopaminérgicos disminuyen el consumo etílico mientras que los antagonistas lo aumentan ${ }^{32}$. Hay que recordar que los estudios de seguimiento de pacientes que sólo reciben un tratamiento de desintoxicación arrojan tasas de recaída del $85 \%{ }^{33}$. Por otro lado, estos resultados son opuestos al ensayo clínico realizado con $50 \mathrm{mgr}$ de amisulpride por dia frente a placebo ${ }^{9}$, en el cual amisulpride a las dosis señaladas no era superior a placebo, y según los autores incluso era inferior a la hora de promover la abstinencia al etanol en pacientes dependientes. Una posible explicación de ese fracaso terapéutico podría ser las diferencias en la posología utilizada en ese estudio frente a la utilizada en el presente estudio ( $50 \mathrm{mgr}$ por día versus $100 \mathrm{mgr}$ por día) ya que hay que recordar que los estudios que polarizan las dosis de amisulpride en prodopaminérgico (50-200 mgr por día) y antidopaminérgico (400-1200 mgr por día) han sido realizados en pacientes diagnosticados de esquizofrenia y no en pacientes con dependencia al alcohol, en los cuales las necesidades posológicas podrían ser otras. Ahondando en el tema podría ser posible que los pacientes con esquizofrenia obtengan respuesta a sus síntomas negativos ya a $50 \mathrm{mgr}$ por día pero que el efecto de esa dosis en pacientes alcohólicos no sea positivo hasta alcanzar los $100 \mathrm{mgr}$ por dia ${ }^{34}$ ya que el estado de hipoactividad dopaminérgica en el cerebro del paciente con dependencia al alcohol que deja de beber es intenso y está mediado por distintas vías neurofisiológicas: reducción de la cantidad y sensibilidad de los receptores D2 sobre todo en el estriado ventral, síntesis de dopamina reducida y reducción en la liberación de dopamina al espacio intersináptico ${ }^{33}$. También es importante destacar que este ensayo previo adolece de dos importantes limitaciones: la muestra es muy pequeña (71 pacientes) y la tasa de abandonos a 6 meses excesivamente elevada (56.3\%) por lo que los resultados obtenidos deberían ser interpretados con cautela. Por otro lado indicar que otros estudios con Amisulpride obtuvieron resultados positivos ${ }^{35}$ en estudios realizados en medios controlados donde se observaba una reducción en el consumo de alcohol. 
Las principales limitaciones de este estudio son: la ausencia de un doble ciego y la existencia de un desfase temporal entre la recogida de datos de los pacientes tratados con Amisulpride que fue posterior a la de los tratados con Naltrexona y Topiramato. No obstante, el hecho de que el entrevistador, el psiquiatra y los terapeutas fueran los mismos en todos los casos y utilizando la misma metodología minimizaría los posibles sesgos.

En resumen, amisulpride a una dosis de $100 \mathrm{mgr}$ por día es igual de eficaz que naltrexona, 50 mgr por día, pero menos que topiramato, 200 mgr por dia, para tratar durante la fase de deshabituación a pacientes diagnosticados de dependencia al alcohol. Esto indicaría la utilidad de amisulpiride, menor que la del topiramato pero igual a la de la naltrexona, en pacientes con dependencia al alcohol tanto en combinación con otros tratamientos, como en pacientes afectados por enfermedad hepática severa, ya que el amisulpride no tiene metabolismo hepático ${ }^{34}$.

\section{AGRADECIMIENTOS}

Este estudio ha sido financiado en parte por el instituto de Salud Carlos III, Centro de Investigación Biomédica en Red de Salud Mental, CIBERSAM.

Todos los autores declaran no tener ningún conflicto de intereses.

\section{REFERENCIAS}

1. Koob GF, Roberts AJ, Schulteis G, Parsons LH, Heyser CJ, Hyytia P et al. Neurocircuitry targets in ethanol reward and dependence. Alcohol Clin Exp Res 1998; 22: 3-9.

2. Koob GF, Le Moal M. Neurobiological mechanisms for opponent motivational processes in addiction. Phil Trans Soc B 2008; 363: 3113-23.

3. Srisurapanot $M_{1}$ JarusuraisinN. Naltrexone for the treatment of alcoholism: a meta-analysis of randomized controlled trials. Int J Neuropsychopharmacol 2005; 8: 267-280.

4. Mason BJ, Ownby RL. Acamprosate for the treatment of alcohol dependence: a review of double - blind, placebo - controlled trials. CNS 2000; 5: 58-69

5. Hughes $\mathrm{J}$, Cook $\mathrm{CCH}$. The efficacy of disulfiram: a review of outcome studies. Addiction 1997; 92: 381-96.

6. Johnson BA, Ait-Daoud N, Bowden CL, DiClemente CC, Roache $J D$, Lawson $\mathrm{K}$ et al. Oral topiramate for treatment of alcohol dependence: a randomised controlled trial. Lancet 2003; 361: 1677-85.

7. Johnson BA, Rosenthal N, Capece JA, Wiegand F, Mao L, Beyers $\mathrm{K}$ et al. Topiramate for treating alcohol dependence: a randomized controlled trial. JAMA 2007; 298: 1641-51.

8. Kampman KM, Pettinati HM, Lynch $K G$, Whittingham $T$, Macfadden W, Dackis C et al. A double-blind, placebo-controlled pilot trial of quetiapine for the treatment of Type A and Type B alcoholism. J Clin Psychopharmacol 2007; 27: 344-51.

9. Marra D, Warot D, Berlin I, Hispard E, Noticies C, Tilikete S. et al. Amisulpride does not prevent relapse in primary alcohol dependence: results of a pilot randomized, placebo-controlled trial. Alcohol Clin Exp Res 2002; 26: 1545-52.

10. World Health Organization. ICD-10, $10^{\text {th }}$ Revision of the International Classification of Diseases. Madrid: World Health Organization; 1992.

11. Rehm J, Room R, Monteiro M, Gmel G, Graham K, Rehn N et al. (2004) Alcohol use. En: Ezzati M, Lopez AD, Rodgers A, Murray CJL editores. Comparative quantification of health risks: Global and regional burden of disease attributable to selected major risk factors, Vol. 1. Ginebra: Organización Mundial de la Salud; 2004. p. 995-1108.

12. Kokkevi $A$, Hartgers $C$. European adaptation of a multidimensional assessment instrument for drug and alcohol dependence. Eur Addict Res 1995;1: 208-10.

13. Sobell LC, Sobell MB. Alcohol timeline followback user's manual. Toronto: Addiction Research Foundation; 1995.

14. Flórez G. Genética y comportamiento: Respuesta al tratamiento de deshabituación alcohólica en función de variables genéticas. Comunicación presentada a las XXXVI Jornadas Nacionales de Socidrogalcohol. Salamanca, 26, 27 y 28 de marzo de 2009.

15. Chang $\mathrm{PH}$, Steinberg MB. Alcohol withdrawal. Med Clin North Am 2001; 85: 1191-12.

16. Volpicelli JR, Pettinati HM, McLellan AT, McLellan T, O'Brien CHP. Enhanced medication and treatment adherence for addiction treatment: the BRENDA model. New York: The Guilford Press; 2001.

17. Anton RF, Moak DH, Latham P. The obsessive compulsive drinking scale: A self-rated instrument for the quantification of thoughts about alcohol and drinking behavior. Alcoholism Clin Exp Res 1995; 19: 92-9.

18. Fagerström KO. Measuring degrees of physical dependence to tobacco smoking with reference to individualization of treatment. Addict Behav 1978; 3: 235-41.

19. Loranger AW, Sartorius N, Andreoli P, Berger P, Buchheim P, Channabasavanna SM et al. The international personality disorder examination. Arch Gen Psychiatry 1994; 51: 215-24.

20. Rollnick S, Heather N, Gold R, Hall W. Development of a short 'readiness to change' questionnaire for use in brief, opportunistic interventions among excessive drinkers. Br J Addict 1992; 87: 743-54.

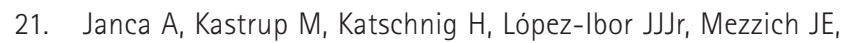
Sartorius N. The World Health Organization Short Disability Assessment Schedule (WHO DAS - S): a tool for the assessment of difficulties in selected areas of functioning of patients with mental disorders. Soc Psychiatr Psychiatr Epidemiol 1996; 31: 349-54.

22. EuroQoL Group. EuroQoL - a new facility for the measurement of health-related quality of life. Health Policy 1990; 16: 199208. 
23. Allen JP, Litten RZ, Strid N, Sillanaukee P. The role of biomarkers in alcoholism medication trials. Alcoholism Clin Exp Res 2001; 25: $1119-25$.

24. Lingjaerd O, Ahlfors UG, Bech P, Dencker SJ, Elgen K. The UKU side effect rating scale. Acta Psychiatr Scand 1987; 76: 1-100.

25. Morisky DE, Green LW, Levine DM. Concurrent and predictive validity of a self-reported measure of medication adherence. Med Care 1986; 24: 67-74.

26. Babor TF, Longabaugh $\mathrm{R}$, Zweben A, Fuller RK, Stout RL, Anton RF et al. Issues in the definition and measurement of drinking outcomes in alcoholism treatment research. J Stud Alcohol 1994; 12: 101-111.

27. Cisler RA, Zweben A. Development of a composite measure for assessing alcohol treatment outcome: operationalization and validation. Alcoholism Clin Exp Res 1999; 23: 263-271.

28. Stout RL. Methodological and statistical considerations in measuring alcohol treatment effects. Alcoholism Clin Exl Res 2003; 27: 1686-1691.

29. Anton RF, O'Malley SS, Ciraulo DA, Cisler RA, Couper D, Donovan DM et al. COMBINE Study Research Group. Combined pharma- cotherapies and behavioral interventions for alcohol dependence - the COMBINE study: a randomized controlled trial. JAMA 2006; 295: 2003-2017.

30. Nutt D. Alcohol and the brain: pharmacological insights for psychiatrists. Br J Psychiatry 1999; 175: 114-19.

31. Koob GF, Le Moal M. Drug abuse: hedonic homeostatic dysregulation. Science 1997; 278: 52-8.

32. Noble EP. Alcoholism and the dopaminergic system. Addict Biol 1996; 1: 333-348.

33. Heinz A, Beck A, Grüsser SM, Grace AA, Wrase J. Identifying the neural circuitry of alcohol craving and relapse vulnerability. Addict Biol 2008; 14: 108-18.

34. Schoemaker $H_{1}$ Claustre $Y$, Fage $D$, Rouquier L, Chergui $K$, Curet 0 et al. Neurochemical characteristics of amisulpride, an atypical dopamine D2/D3 receptor antagonist with both presynaptic and limbic selectivity. J Pharmacol Exp Ther 1997; 280: 83-97.

35. Hermann D, Smolka MN, Wrase J, Klein S, Nikitopoulos J, Georgi A et al. Blockade of cue-induced brain activation of abstinent alcoholics by a single administration of amisulpride as measured with fMRI. Alcohol Clin Exp Res 2006; 30: 1349-54. 